\title{
Supporting Phase Stability on Interconnected Grids by Synchronous Renewable Virtual Power Plants
}

\author{
Mostafa Abdollahi, Jose Ignacio Candela, Joan Rocabert, Raul Santiago Munoz Aguilar, and Juan Ramon Hermoso \\ Technical University of Catalonia \\ Barcelona, Spain \\ Mostafa.Abdollahi@upc.edu
}

\begin{abstract}
Rapid growing on the power level of renewable generation units leads to that using more adaptable and flexible control techniques in this units becomes more important for grid operators. In this paper, after introducing Renewable Static Synchronous Generation Units (RSSGU) as units with flexible dynamics capability, forming of renewable Virtual Power Plants based on this RSSGUs (VPP-SSG) is suggested as a solution for overcoming phase stability challenges on interconnected generation areas. Based on the dynamic modeling and small signal analysis, an algorithm is presented for the dynamic designing of VPP-SSGs aims to provide supporting damping for both local and interarea oscillatory modes. Modal analysis and time domain study on active powers inside of generation areas and tie lines on two area system using Simulink confirms that these type of VPPSSGs can support phase stability on power grid with interconnected generation areas.
\end{abstract}

Keywords-Renewable static synchronous generation units; Synchronous power controller; Dynamic modeling; Damping oscillatory modes; Phase stability.

\section{INTRODUCTION}

Research studies on international organizations show almost $90 \%$ of new generation capacity installed in Europe is coming from renewables in 2016 [1]. This means Renewable Generation Units (RGU) with several type resources are getting higher power level and in this way they will play more remarkable role in supporting of power grids. In this situation, having control technology more adaptable with the other Classic Generation Units (CGU) would be more acceptable for these RGUs in the grid. Nowadays by introducing Virtual Power Plant (VPP) based on these RGUs, this control acclimation will be more important, because a VPP equipped with proper control technology can expand RGUs capabilities to the transmission level then to the whole of the grid, so in this case RGUs will support CGUs more than before. In the practical, this means grid operators not only on the low level even on the higher level can enlist these renewable VPP capabilities to overcome grid challenges [2] Phase stability is one of these critical challenges on interconnected generation areas, and it will be more challengeable when the perspective of the grid is much expanded such as European Super Grid [3]. Recently a few control strategies are introduced for renewables to cope with both local and interarea phase stability, which almost all of them tried to provide required dynamics for phase supports. Some of them are an emulation of Synchronous Generation (SG) dynamics [4], and sometimes this technique is merged with the HVDC technologies [5]. One of introduced methods for controlling the renewables is Synchronous Power Controller (SPC) which is simple in application and has high level of adaptivity with the rest of the grid on large scale [6]. As a background of this work, previously on [7] and [8], it has been shown the SPC technique can be expanded for renewables as a Static Synchronous Generators (SSG) for support the voltage and phase stability on a long AC transmission system. Based on this background in this paper, we are following two main goals. Firstly, introducing a Renewable Static Synchronous Generation Unit (RSSGU) based on the SPC then the discussion about the formation of Renewable VPPs based on these RSSGUs (VPPSSG). Secondly, using dynamic capabilities of these VPP-SSGs as a solution for supporting the phase stability from the local and interarea point of view. So in the following, firstly a summary of RSSGU and dynamic flexibility on its output active power is presented, then the formation of VPP-SSGs is discussed shortly. After that, dynamic modeling of power grid in presence of VPPSSG is done aims to extract small signal model of the whole of the grid. Then, based on this modeling, a simple algorithm is proposed for targeting this dynamic design on the providing supplementary damping for both local and inter area oscillatory modes. Finally, by applying this algorithm, doing modal analysis and time domain test on a VPP-SSGs connected to Kundur system, this solution is evaluated for phase stability support.

\section{STATIC SYNCHRONOUS GENERATION}

\section{A. Renewable Static Synchronous Generation Units(RSSGU)}

As mentioned in previous works [7,8], a Voltage Source Converter (VSC) in the renewable SSG unit can be controlled as depicted in Fig. 1. The main idea is having flexible dynamic characteristics on this RSSGU aim to interact with other CGUs properly. This flexible interaction is more important where RSSGU has big generation capacity. Incorporating Virtual Admittance (VA) technique and Electro Mechanical (EM) model makes this dynamic flexibility for VSC. By implementing this technique, active power of RSSGU will have flexible dynamics and complies the control loop as drawn in Fig. 2. As can be seen, by direct measuring of active power, the grid frequency $(\Delta \omega)$, phase angle $(\theta)$ and required power angle $(\delta)$ will be extracted, receptively. Finally, this $\delta$ will generate the output active power of RSSGU. Having at least two-degree freedom in the dynamics of this control loop, means controllable virtual damping $(D)$ and virtual inertia $(H)$, provides this flexibility for each RSSGU such as sample tests are shown in Fig. 3. These tests confirm that each RSSGU can be designed, 
controlled and tuned for satisfying different goals of the grid operators. One of this goal can be supporting stability in the grid by providing supplementary damping in generation areas and/or on interarea tie lines.

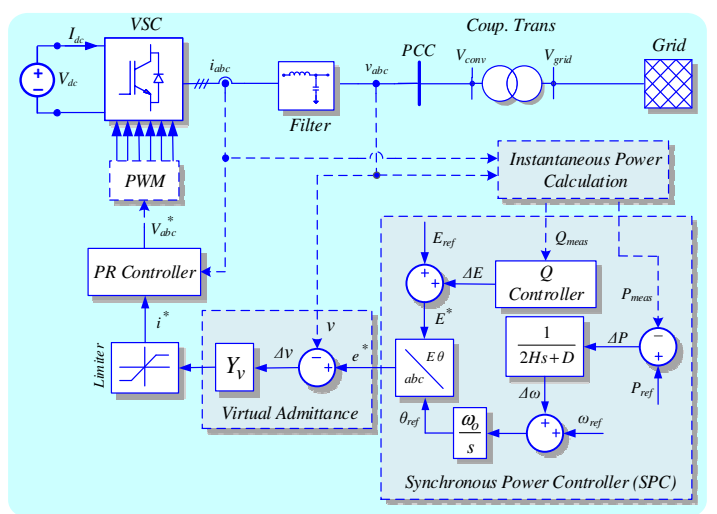

Fig. 1. Control of a Renewable Static Synchronous Generation Unit

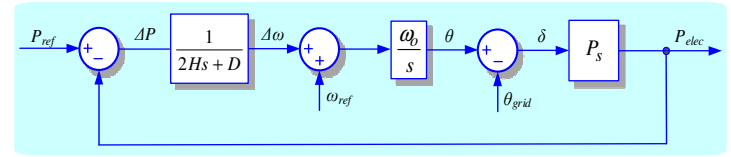

Fig. 2. Dynamic model of a RSSGU
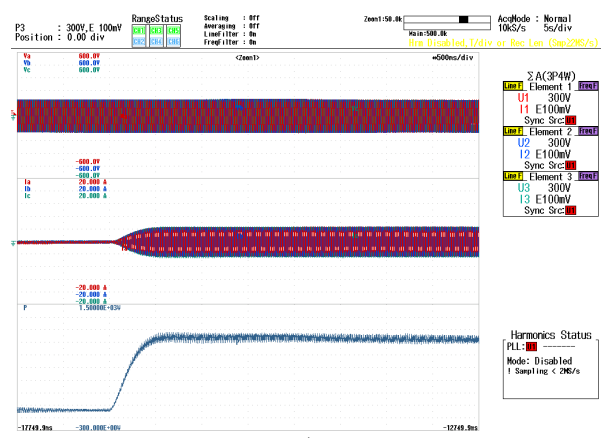

a)

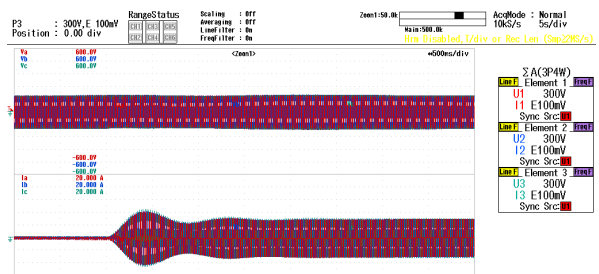

3:i:

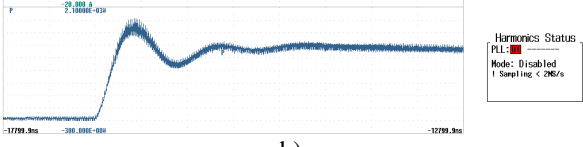

b)

Fig. 3. Dynamic flexibility in active power of RSSGU, a) high power damping, b) moderate power damping

B. Renewable Virtual Power Plant Based on SSG (VPP-SSG)

Simplicity in structure and implementation is one of the important benefits of RSSGUs introduced in the last section. The very high similarity with the classic synchronous generation units is the reason of this simplicity. This property can be a positive sign for expanding this technique on the control of a set of converters with several type of renewable source. By increasing in the power level of RSSGUs then the emergence of VPP idea, the control technique implemented on RSSGUs can be expanded for a VPP-SSG. A simple structure of VPP-SSG can be as shown in Fig. 4. A VPP-SSG is combined of several RSSGUs with different renewable sources which are operated under a Control Center. For providing a unique dynamics characteristics from VPP-SSG side to grid, each RSSGU will receive specific control signal according to its own terms and features. Finally, by applying this coordinated and global control on RSSGUs, the VPP-SSG can be controlled for predefined goals. One of these goals can be supporting phase stability in transmission level which is discussed in the next sections.

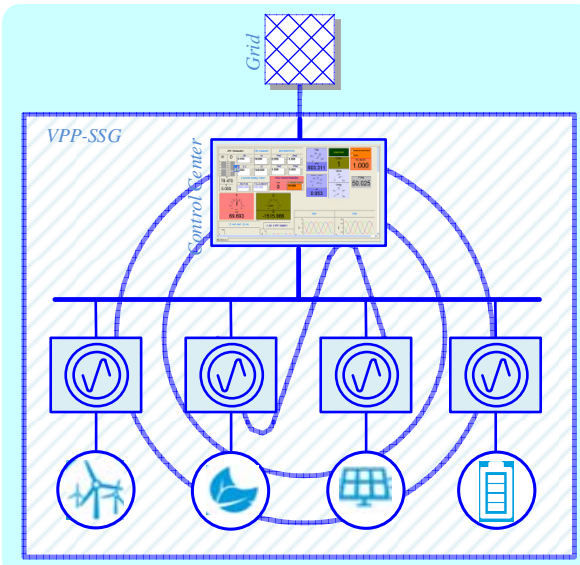

Fig. 4. Operation of Renewable Virtual Power Plant based on RSSG units

\section{DYNAMIC MODELING OF VPP-SSG ON POWER GRID}

In this section, modeling and design of VPP-SSG on power grid will be discussed. The general pattern is similar to methods which are presented on basic resources [9]. By integration of several RSSGUs together and forming a VPP-SSG with high power level, as well as regard to the emulation of SG characteristics in each RSSGUs and therefore on the whole of the VPP-SSG, the Thevenin equivalent model can be used for electrical modeling of a VPP-SSG in the power grid, similar to the modeling of other CGUs. So, in a power grid with two type of buses: PV and PQ as depicted in Fig. 5, a VPP-SSG can be considered as power supply connected to a PV bus such as other CGUs. Process of dynamic modeling for the power grid in presence of VPP-SSG aims to extract state space model of the whole of the system can be summarized as a flowchart shown in Fig. 6. As can be seen, after introducing the specific data of understudy system (grid, CGUs, VPP-SSG), the initial operating point of the system can be defined by doing power flow. In continue, Algebraic Equation of Power Grid (AEPG) will be linearized where can be collected as (1) and then can be rewritten as (2). In this set of equations, by finding constant coefficient $\left(A_{2}, \ldots, D_{2}\right)$ the linear relationship between magnitude-angle of voltages on PV $\left(\Delta V_{g i} \angle \Delta \theta_{g i}\right)$ as well as on PQ $\left(\Delta V_{L j} \angle \Delta \theta_{L j}\right)$ buses with the power angle $\left(\Delta \delta_{i}\right)$ in generation units will be determined. We will see that the $A_{2}$ and $C_{2}$ are two important constant coefficients that will create a link between AEPG and Dynamic Equation of Generation Units (DEGU). In the next 
step, DEGU will be linearized and a set of the equation will be extracted such as (3). The main goal in this set is creating a linear link between dynamic characteristics and electrical features of each generation units including VPP-SSG.

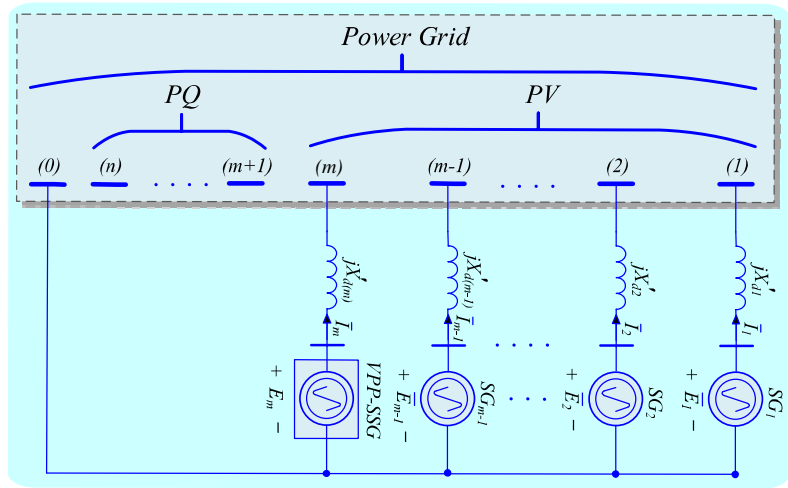

Fig. 5. Modeling of Power Grid with VPP-SSG

$$
\begin{gathered}
{\left[\begin{array}{ll}
A_{1} & B_{1} \\
R_{1} & S_{1} \\
C_{1} & D_{1} \\
U_{1} & V_{1}
\end{array}\right]\left[\begin{array}{l}
\Delta V_{g} \\
\Delta V_{L} \\
\Delta \theta_{g} \\
\Delta \theta_{L}
\end{array}\right]=\left[\begin{array}{c}
G \\
\Phi_{1} \\
H \\
\Phi_{2}
\end{array}\right][\Delta \delta]} \\
{\left[\begin{array}{l}
\Delta V_{g} \\
\Delta V_{L} \\
\Delta \theta_{g} \\
\Delta \theta_{L}
\end{array}\right]=\left[\begin{array}{l}
A_{2} \\
B_{2} \\
C_{2} \\
D_{2}
\end{array}\right][\Delta \delta]}
\end{gathered}
$$

Moreover, in this set the $\left(\Delta \boldsymbol{P}_{e}\right)$ is the linearized format of the electric output power of generation units based on the power angle $(\Delta \boldsymbol{\delta})$, where the $A_{2}$ and $C_{2}$ appear for creating this linear link. So, in (3) the effect of grid parameters is accounted into dynamic modeling of generation units, and AEPG and DEGU as two sets of main equations are combined. The base three constants include $\boldsymbol{K}_{\mathbf{1}}, \boldsymbol{K}_{\mathbf{2}}$ and $\boldsymbol{K}_{\mathbf{3}}$ will be obtained during linearization process of $\Delta \boldsymbol{P}_{\boldsymbol{e}}$ as explained on (4). The merging two parts of equation (3) will lead to set (5) where DEGU is rewritten again. As can be seen, the matrix of generation units inertia $\left(\boldsymbol{H}_{\boldsymbol{M}}\right)$ appears inversely on $\boldsymbol{K}_{\mathbf{4}}$ while both damping $\left(\boldsymbol{D}_{\boldsymbol{M}}\right)$ and inertia $\left(\boldsymbol{H}_{\boldsymbol{M}}\right)$ matrixes will create the $\boldsymbol{K}_{\mathbf{5}}$. Here we can see the effect of the available two dynamic freedom degrees on the VPP-SSG $\left(D_{S S G}, H_{S S G}\right)$ which will affect the dynamic model of the power grid. In the next step, by defining $\Delta \boldsymbol{\delta}$ and $\Delta \boldsymbol{\omega}$ as dynamic states of grid, the state space model of the system can be summarized as (6). Where, $\boldsymbol{F}$ is identity matrix for making the link between states and corresponding derivate, $\boldsymbol{A}$ is the state matrix and $\boldsymbol{O}$ is the zero matrix with proper dimension. The modes of $\boldsymbol{A}$ will give us the condition of small signal stability on the whole of the system which can be used to design and tune of the VPP-SSG based on the modal analysis technique aims to meet the specific requirements. One of the applicable strategies in the dynamic design of VPP-SSG can be subjected to the assessment of supplementary damping ratio provided by the VPP-SSG in grids, such as presented in Fig. 7. In this case, the clear goal can be the analysis of damping ratio in local and interarea modes of the grid where all classics damping provider such as Power System Stabilizer (PSS) are deactivated. Based on this algorithm we can consider a primary value for $H_{S S G}$ in the typical range for VPP-SSG. Aim to have better dynamic performance as well as avoiding unexpected dynamic change in the base system condition, this value can be close to the inertia of the neighbor generation units but it is suggested to be smaller than them.

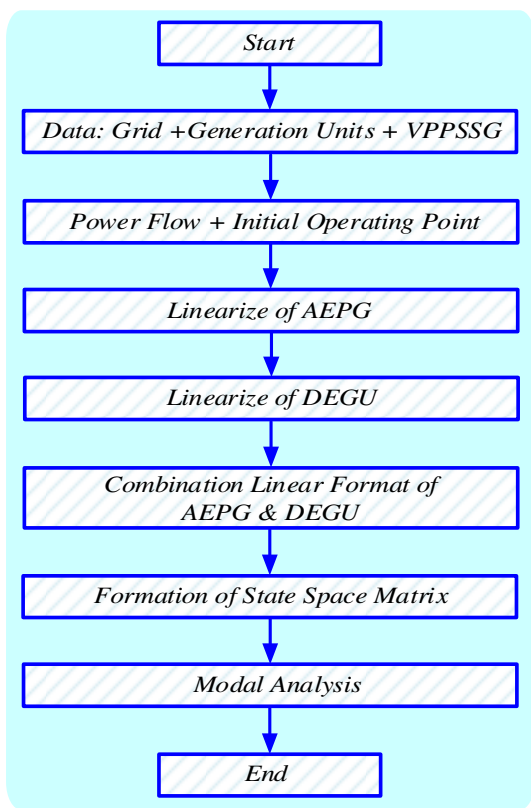

Fig. 6. Steps in Dynamic Modeling Process

$$
\left\{\begin{array}{l}
\frac{2}{\omega_{s}} H_{M} \frac{d}{d t} \Delta \omega=-\Delta P_{e}-D_{M} \Delta \omega \\
\Delta P_{e}=\left(K_{1}+K_{2} C_{2}+K_{3} A_{2}\right) \Delta \delta
\end{array}\right.
$$

$$
\begin{aligned}
& \left\{\begin{array}{c}
\boldsymbol{K}_{\mathbf{1}}=\operatorname{diag}\left(k_{11}, k_{12}, \ldots \ldots k_{1 m}\right) \\
\boldsymbol{K}_{2}=\operatorname{diag}\left(k_{21}, k_{22}, \ldots \ldots k_{2 m}\right) \\
\boldsymbol{K}_{\mathbf{3}}=\operatorname{diag}\left(k_{31}, k_{32}, \ldots \ldots k_{3 m}\right) \\
k_{1 i}=\left.\frac{\partial P_{e i}}{\partial \delta_{i}}\right|_{\theta_{i}, V_{i}=c t e}=\frac{E_{i} V_{i}}{x_{d i}^{\prime}} \cos \left(\delta_{i}-\theta_{i}\right) \\
k_{2 i}=\left.\frac{\partial P_{e i}}{\partial \theta_{i}}\right|_{\delta_{i}, V_{i}=c t e}=-\frac{E_{i} V_{i}}{x_{d i}^{\prime}} \cos \left(\delta_{i}-\theta_{i}\right) \\
k_{3 i}=\left.\frac{\partial P_{e i}}{\partial V_{i}}\right|_{\delta_{i}, \theta_{i}=c t e}=\frac{E_{i}}{x_{d i}^{\prime}} \sin \left(\delta_{i}-\theta_{i}\right)
\end{array}\right. \\
& \left\{\begin{array}{c}
\frac{\boldsymbol{d}}{\boldsymbol{d} \boldsymbol{t}} \Delta \boldsymbol{\omega}=\boldsymbol{K}_{\mathbf{4}} \Delta \boldsymbol{\delta}+\boldsymbol{K}_{\mathbf{5}} \Delta \boldsymbol{\omega} \\
\boldsymbol{K}_{\mathbf{4}}=-\frac{\boldsymbol{\omega}_{s}}{\mathbf{2}} \boldsymbol{H}_{\boldsymbol{M}}^{-\mathbf{1}}\left(\boldsymbol{K}_{\mathbf{1}}+\boldsymbol{K}_{\mathbf{2}} \boldsymbol{C}_{\mathbf{2}}+\boldsymbol{K}_{\mathbf{3}} \boldsymbol{A}_{\mathbf{2}}\right) \\
\boldsymbol{K}_{\mathbf{5}}=-\frac{\boldsymbol{\omega}_{s}}{\mathbf{2}} \boldsymbol{H}_{\boldsymbol{M}}^{-\mathbf{1}} \boldsymbol{D}_{\boldsymbol{M}} \\
\boldsymbol{D}_{\boldsymbol{M}}=\operatorname{diag}\left(D_{1}, \ldots, D_{S S G}\right) \\
\boldsymbol{H}_{\boldsymbol{M}}=\operatorname{diag}\left(H_{1}, \ldots, H_{S S G}\right)
\end{array}\right.
\end{aligned}
$$

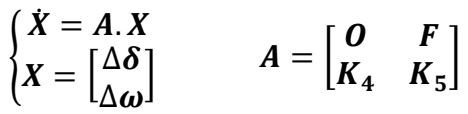


After that, by selecting a small value for $D_{S S G}$ (where damping effect of all classic units is still close to zero), we can start dynamic modeling process as described on Fig. 6. By having state space model then extracting all eigenvalues and eigenvectors of $\boldsymbol{A}$, all local and interarea oscillatory modes of the system can be accessible. Now, if current condition of system is satisfying the predefined requirement in damping ratio $(\xi)$ so this selected values can be good suggestion for tuning on VPP-SSG. But if not, before any extra action, first the condition of internal damping ratio ( $\xi_{S S G}^{\text {internal }}$ ) on VPP-SSG should be investigated. If we didn't cross the border of fully damped condition inside of the VPP-SSG $\left(\xi_{S S G}^{\text {internal }}<1\right.$ ), we can select a bigger value for $D_{S S G}$ and in this way we can go closer to the desired zeta for modes $(\xi)$. If VPP-SSG was fully damped internally, this means the selected value for $H_{S S G}$ was not enough big and our VPP-SSG has very weak dynamic compare to other part of system. In this case, solution is the suggestion of a new bigger $H_{S S G}$ to avoiding this early fully damping condition and after that continuing to define new values for $D_{S S G}$. This iteration process can be continued until arriving to a condition which is satisfying the desired damping ratio $(\xi)$ in each local or interarea (or both) oscillatory modes. This simple iteration algorithm can be used for two important goals: a) design of global parameters on a VPP-SSG, b) design and tune of each RSSGU inside of the VPP-SSG. In the first case, the purpose can be satisfying zeta on oscillatory modes of an external grid $(\xi)$, and in this way we are calculating the equal $D_{S S G}$ and $H_{S S G}$ for tuning on VPP-SSG. But in the second case, the main objective is satisfying an equivalent internal zeta on VPP-SSG ( $\xi_{S S G}^{\text {internal }}$ ) and we are tuning each RSSGUs on the specific value of $D_{S S G(i)}$ and $H_{S S G(i)}$. In simple word, in this case, we are designing each RSSGUs to get a specific unique dynamic behavior on the whole of the VPP-SSG.

\section{VPP-SSG ON TwO AREA SYSTEM}

\section{A. Base System Analysis}

Schematic diagram of base kundur system is shown in Fig. 8. As can be seen, this system contains two generation areas which are connected through a long tie line. There are two 900MW CGUs in each area which are loading around $750 \mathrm{MW}$. Moreover, there is a compact local load in each area (L7=967MW, L9=1767MW) which are modeled as constant impedance type. Surplus generation of Area I (400-450MW) is transferred to Area II by line for supporting L9. All classic damping provider such as PSS considered to be deactivated to can have more clear view about the stability condition on base system. Aim to have semi flat initial operating point in time domain analysis, just a small value is considered for damping constant $\left(K_{\mathrm{d}}=0.01 \mathrm{pu}\right)$ in each generation units. Small signal modeling is done by using method presented before, then all dynamic modes are extracted by doing the modal analysis. Participation Factor Matrix (PFM) of generation units in modes are calculated thus categorization of modes is possible in this way. Results are shown in Fig. 9 and reported in Table I. As can be seen, each area has a local mode with frequency slightly higher than $1 \mathrm{~Hz}\left(\lambda_{1,2}=1.14 \mathrm{~Hz}, \lambda_{7,8}=1.17 \mathrm{~Hz}\right)$ and there is an interarea mode around half of hertz $\left(\lambda_{5,6}=0.44 \mathrm{~Hz}\right)$. All of these modes have small damping ratio (5\% and less) which is coming from the primary assumption $\left(K_{\mathrm{d}}=0.01 \mathrm{pu}\right)$. For validation of dynamic modeling results, a discrete time domain modeling is done in Simulink. Fig. 10 shows disturbances in active power of generation units where a $40 \mathrm{MW}$ load is out from base load L9. Because load outage happened on Area II, this disturbance will dispatched through the tie line (see Fig. 16) and the $S G_{1: 2}$, where located on Area I, will face disturbance with the frequency equal to interarea oscillation. This mode has around 5\% damping ratio so it will appear for more than 10 seconds, where dashed plot on Fig. 16 also confirms it. But in Area II, because the disturbance is a local turbulence, as well as a part of the power is coming from Area I through the tie line and there is a reaction on Area I, so both local and interarea modes will appear on power disturbances in this area (see $P_{S G 3: 4}$ in Fig. 10).

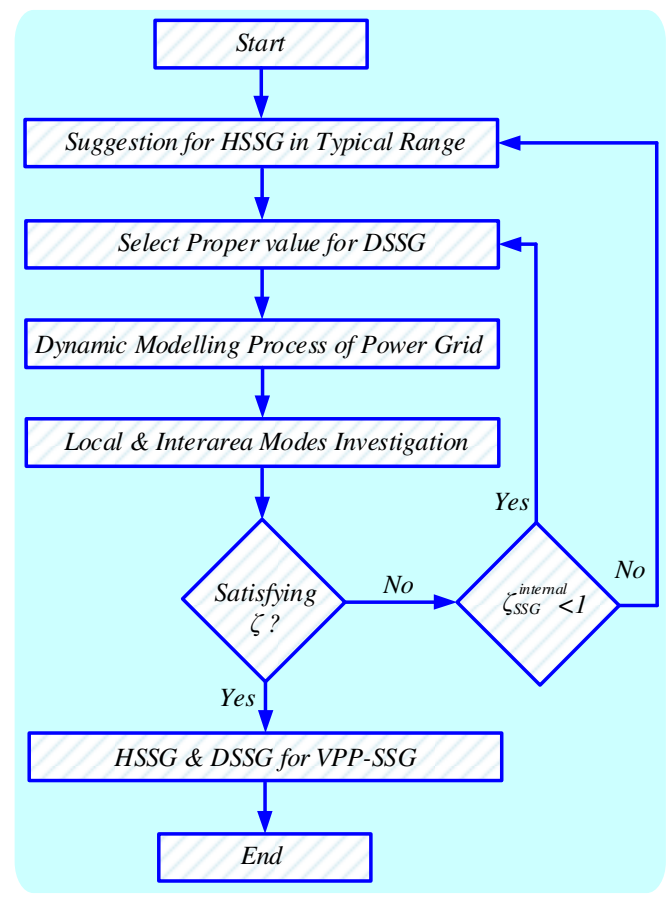

Fig. 7. Algorithm for Design of Dynamic Parameters in a VPP-SSG

\section{B. VPP-SSG Connected to Two Area System}

One possibility for VPP-SSG connection is shown in Fig. 11, where $S G_{2}$ is replaced with the VPP-SSG. This case can be useful for investigation of VPP-SSG effects when it is installed on the sending power areas. Modal analysis results are show on Fig. 12 till Fig. 14, where VPP-SSG is designed and tuned based on the technique presented on Fig. 7, and condition of rest of CGUs is same as base system. Impact of VPP-SSG's virtual damping flexibility on oscillatory modes is shown in Fig. 12 for $H_{S S G}=$ $2.94 s, 0 \leq D_{S S G} \leq 0.44 p u$. By selecting a primary $H_{S S G}$ close to base system (a bit smaller) primary frequency of local mode (I) $\left(\lambda_{1,2}\right)$ is slightly higher than the base case. By increasing on $D_{S S G}$, the $\lambda_{1,2}$ will move to more stable zones and the frequency of mode decreases to lesser quantities. This pattern will continue in order to the maximum damping ratio on $\lambda_{1,2}$ will happen $\left(\xi_{\max }=0.364 \mathrm{pu}\right)$ on frequency around of local frequency of base system in this area. After that, by increasing more $D_{S S G}$ this local mode comes back to zones with smaller stability margin 
and during whole of this process, frequency is still decreasing. At the same time of changing on $\lambda_{1,2}$, interarea mode $\left(\lambda_{5,6}\right)$ as a dependent variable to VPP-SSG, will also get more damping linearly and on the break point of $\lambda_{1,2}$, it has $\xi_{\text {inter }}=0.223 p u$. Because all generation units are participating on $\lambda_{5,6}$ so the slope of frequency reducing is very smaller than $\lambda_{1,2}$ case. Although from interarea mode $\left(\lambda_{5,6}\right)$ point of view, having bigger $D_{S S G}$ can be better condition but from local mode point of view $\left(\lambda_{1,2}\right)$ this condition cannot be applicable and is not safe.

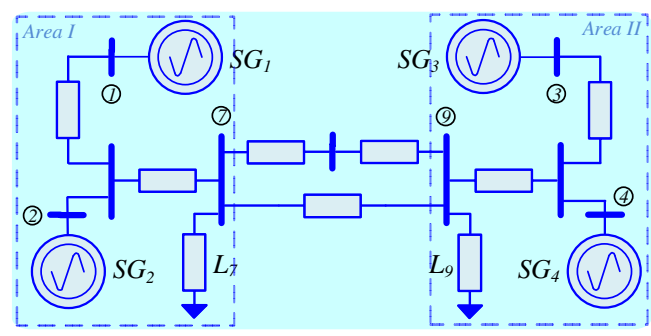

Fig. 8. Two Area System with full Classic Generation Units.

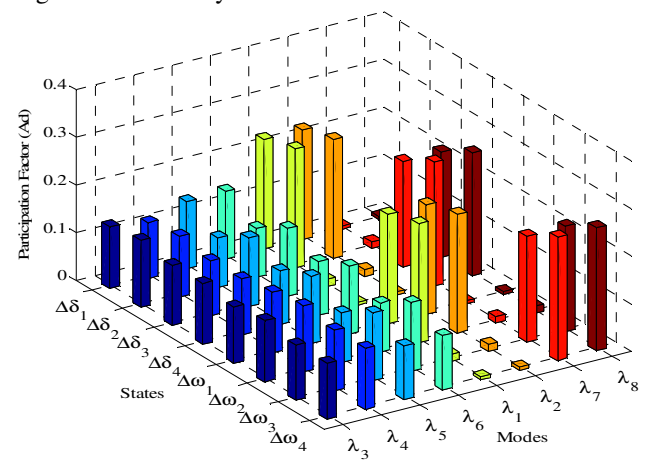

Fig. 9. Participation Factor of Classic Generation Units

\begin{tabular}{|c|c|c|c|c|c|}
\multicolumn{7}{c|}{ Table I. Oscillatory modes of base system } \\
\hline $\boldsymbol{\lambda}$ & Eige & $\boldsymbol{f}(\mathbf{H z})$ & $\xi$ & states & Description \\
\hline$\lambda_{\mathbf{1 , 2}}$ & $\begin{array}{l}-0.1453 \\
\pm 7.1776 i\end{array}$ & 1.1423 & 0.0202 & $\Delta \delta_{1: 2}, \Delta \omega_{1: 2}$ & Local mode I \\
\hline$\lambda_{\mathbf{3}, \mathbf{4}}$ & -3.4898 & - & 1.0000 & - & - \\
\hline$\lambda_{\mathbf{5 , 6}}$ & $\begin{array}{l}-0.1489 \\
\pm 2.8128 i\end{array}$ & 0.4477 & 0.0529 & $\Delta \delta_{1: 4}, \Delta \omega_{1: 4}$ & $\begin{array}{c}\text { Inter area } \\
\text { mode }\end{array}$ \\
\hline$\lambda_{\mathbf{7 , 8}}$ & $\begin{array}{l}-0.1523 \\
\pm 7.3653 i\end{array}$ & 1.1722 & 0.0207 & $\Delta \delta_{3: 4}, \Delta \omega_{3: 4}$ & Local mode II \\
\hline
\end{tabular}

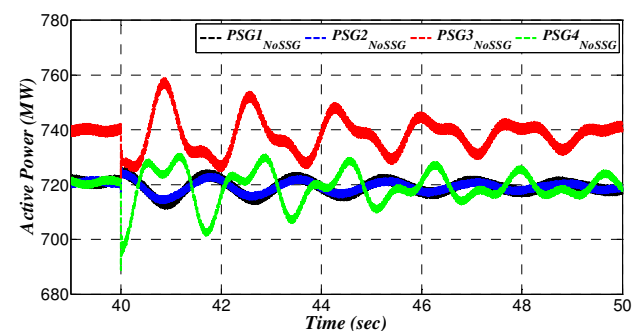

Fig. 10. Disturbances in Active Power of Generation Units in Base System

Moreover, as can be seen in Fig. 12, the increasing on $D_{S S G}$ cannot have any effect on the neighbor local modes $\left(\lambda_{7,8}\right)$. It should be noted that by attention to the PFM on base system (see Fig. 9), this decoupling of local modes in each area from generation units on the neighbor area was not unexpected.

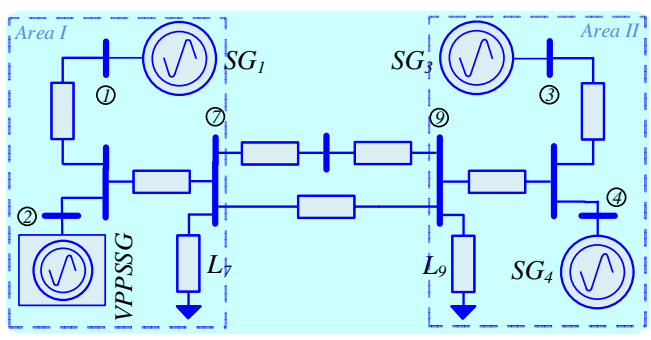

Fig. 11. VPP-SSG in Two Area System

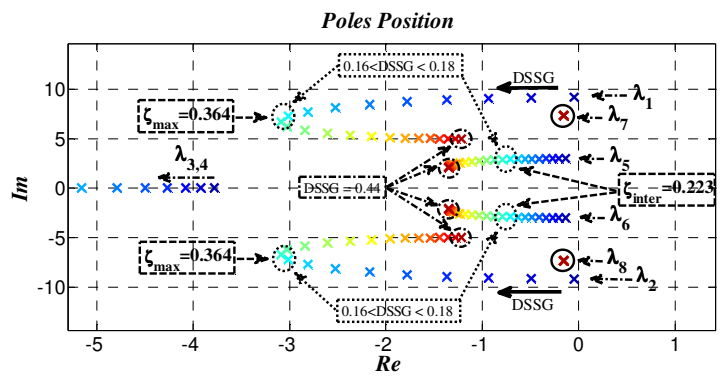

Fig. 12. Impact of VPP-SSG on Local \& Interarea Oscillatory Modes

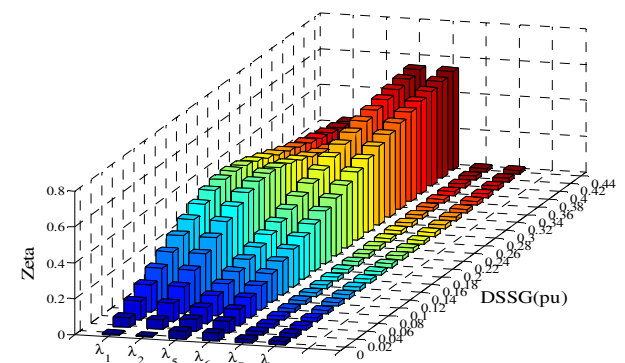

Fig. 13. Damping Support of Oscillatory Modes by VPP-SSG.

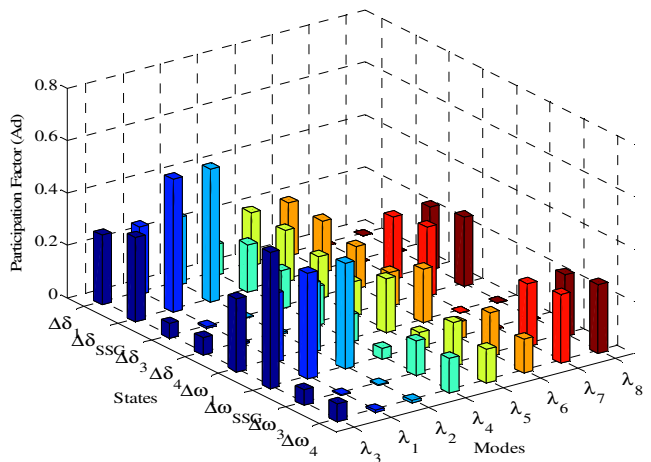

Fig. 14. Participation Factor of VPP-SSG in System Modes

Supplementary damping in all oscillatory modes provided by VPP-SSG is plotted in Fig. 13. Based on the results shown in Fig. 12 and Fig. 13 we can say for pre-selected $H_{S S G}=2.94 \mathrm{~s}$, selecting $D_{S S G}=0.16 p u$ can be an optimal tune for support enough both local and interarea modes. PFM and modal analysis results of this optimal tuning on VPP-SSG are reported in Fig. 14 and Table II. Comparing Fig. 9 and Fig. 14 shows the participation factor of VPP-SSG on $\lambda_{1,2}$ is reinforced and this confirms the new supplementary damping is controlled directly by increasing on $D_{S S G}$. Therefore, zeta of $\lambda_{1,2}$ is increased from 
$\xi_{\lambda_{1,2}}=0.0202 p u$ in base case to remarkable value $\xi_{\lambda_{1,2}}=$ $0.364 p u$ on the optimal tuning of VPP-SSG (compare Table I and Table II). Moreover, although in the base system we can see an almost uniform participation of all units in interarea mode $\lambda_{5,6}$ (see Fig. 9), but after having big $D_{S S G}$ on VPP-SSG and tune it on optimal operation point, this uniformity is nearly gone and now generation units of Area I, especially VPP-SSG, has higher factor on this mode. This confirms that $\lambda_{5,6}$ is affected indirectly from $D_{S S G}$ which leads to change damping ratio from $\left(\xi_{\lambda_{5,6}}=\right.$ $0.0529 p u)$ to four times bigger in presence of VPP-SSG $\left(\xi_{\lambda_{5,6}}=0.223 p u\right)$. Similar to the base case, still only local generation units of Area II has participated on $\lambda_{7,8}$ (compare Fig. 9 and Fig. 14), so we don't expect that VPP-SSG has any effect on this neighbor local mode.

Table II. Oscillatory modes in presence of VPP-SSG

\begin{tabular}{|c|c|c|c|c|c|}
\hline $\boldsymbol{\lambda}$ & Eige & $\boldsymbol{f}(\mathrm{Hz})$ & $\boldsymbol{\xi}$ & states & Description \\
\hline$\lambda_{\mathbf{1 , 2}}$ & $\begin{array}{l}-2.9245 \\
\pm 7.4827 i\end{array}$ & 1.1909 & 0.3640 & $\Delta \delta_{1, S S G}, \Delta \omega_{1, S S G}$ & Local mode I \\
\hline$\lambda_{\mathbf{3}, \mathbf{4}}$ & -5.9562 & - & 1.0000 & - & - \\
\hline$\lambda_{\mathbf{5 , 6}}$ & $\begin{array}{l}-0.6684 \\
\pm 2.9182 i\end{array}$ & 0.4644 & 0.2233 & $\Delta \delta_{1: 4}, \Delta \omega_{1: 4}$ & $\begin{array}{c}\text { Interarea } \\
\text { mode }\end{array}$ \\
\hline$\lambda_{\mathbf{7 , 8}}$ & $\begin{array}{l}-0.1535 \\
\pm 7.3552 i\end{array}$ & 1.1706 & 0.0209 & $\Delta \delta_{3: 4}, \Delta \omega_{3: 4}$ & Local mode II \\
\hline
\end{tabular}

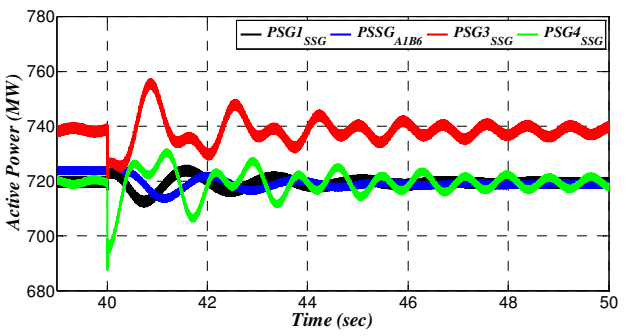

Fig. 15. Restoration of Active Power Disturbances by VPP-SSG

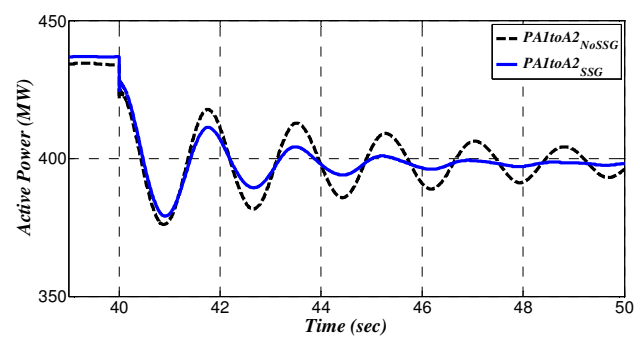

Fig. 16. Surplus Damping in Interarea Power

Same scenario on time domain analysis is repeated for system with VPP-SSG when is tuned on this optimal condition and results are presented on Fig. 15 and Fig. 16. Similar to the base system, here Area I is also affected only by interarea oscillation but now this disturbance is damped compared to the base case (see Fig. 16). More lasting oscillation on $P_{S G 3: 4}$ reaffirms that VPP-SSG cannot have the effect on neighbor area's local mode. Most different between $P_{S G 3: 4}$ in Fig. 15 and Fig. 10 is because of that, on base case (Fig. 10) both $\lambda_{7,8}$ and $\lambda_{5,6}$ have very small damping ratio, so there is a combination of local and interarea oscillations on this powers; but in supported system by VPP-SSG, the $\lambda_{5,6}$ will damped fast (less than 6 seconds) and just $\lambda_{7,8}$ will remain for longer time. So $P_{S G 3: 4}$ have single frequency oscillation close to this local mode $\left(\lambda_{7,8}\right)$ at the end part of the simulation time.

\section{CONCLUSION}

In this work, after presenting several parts of the control system in a RSSGU, it was shown that EM part of SPC can provide a flexible dynamics for output active power of RSSGU. The formation of VPPs based on the RSSGUs is introduced and discussed that by proper tuning of each RSSGUs, the whole of the VPP-SSG can have unique dynamic characteristics thus RSSGUs flexibility can be generalized on transmission level. Then, it is suggested that VPP-SSG's capability can be used by grid operators aims to support stability in the power grids by providing supplementary damping for local and interarea oscillatory modes. By using the detailed dynamic model of the power grid in presence of VPP-SSG and extracting state space model of the whole of the system, it was shown that dynamic parameters of VPP-SSG $\left(D_{S S G}, H_{S S G}\right)$ can have key roles on the condition of system modes. Moreover, a simple primary algorithm is introduced for targeting of this dynamic design on damping oscillatory modes. Testing of VPP-SSG on Kundur system by doing modal analysis using root-locus index and participation factor matrix; as well as time domain simulations confirmed that formation of these type of VPPs on generation areas not only can damp local modes on installation area but also can improve damping of interarea power oscillation on interconnected areas.

\section{ACKNOWLEDGMENT}

This work was supported by the Spanish Ministry of Science under Project ENE2013-48428-C02-2-R.

\section{REFERENCES}

[1] Renewables 2016 Global Status Report, REN21. 2016. availiable on: "http://www.ren21.net/wpcontent/uploads/2016/06/GSR_2016_Full_Re port.pdf'.

[2] B. Kroposki et al., "Achieving a 100\% Renewable Grid: Operating Electric Power Systems with Extremely High Levels of Variable Renewable Energy," in IEEE Power and Energy Magazine, vol. 15, no. 2, pp. 61-73, March-April 2017.

[3] "Research and development plan-European grid towards 2020 challenges and beyond," Brussels, Belgium, Tech. Rep., Entso-E, 2010.

[4] W. Zhang, A. M. Cantarellas, J. Rocabert, A. Luna and P. Rodriguez, "Synchronous Power Controller With Flexible Droop Characteristics for Renewable Power Generation Systems," in IEEE Transactions on Sustainable Energy, vol. 7, no. 4, pp. 1572-1582, Oct. 2016.

[5] E. Rakhshani, D. Remon, A. M. Cantarellas, J. M. Garcia, P. Rodriguez, "Virtual Synchronous Power Strategy for Multiple HVDC Interconnections of Multi-Area AGC Power Systems," in IEEE Transactions on Power Systems , vol.PP, no.99, pp.1-1, 2016.

[6] D. Remon, A. M. Cantarellas, P. Rodriguez, "Equivalent Model of LargeScale Synchronous Photovoltaic Power Plants," in IEEE Transactions on Industry Applications, vol. 52, no. 6, pp. 5029-5040, Nov.-Dec. 2016.

[7] M. Abdollahi, J. I. Candela, J. Rocabert, R. S. M. Aguilar and J. R. Hermoso, "Improving long line stability by integrating renewables using static synchronous generators," 2016 IEEE International Conference on Renewable Energy Research and Applications (ICRERA), Birmingham, pp. 512-517, 2016.

[8] M. Abdollahi, J. I. Candela, J. Rocabert, R. S. M. Aguilar and J. R. Hermoso, "Synchronous power controller merits for dynamic stability improvement in long line by renewables," 2016 IEEE International Conference on Renewable Energy Research and Applications (ICRERA), Birmingham, pp. 760-765, 2016.

[9] P. Kundur, 'Power System Stability and Control', N.Y: M-Hil, 1994. 
